\title{
Ccreative
commons
}

ISSN 1855-3966 (printed edn.), ISSN 1855-3974 (electronic edn.)

\author{
ARS MATHEMATICA CONTEMPORANEA 17 (2019) 617-626 \\ https://doi.org/10.26493/1855-3974.1891.3b7 \\ (Also available at http://amc-journal.eu)
}

\section{On flag-transitive automorphism groups of symmetric designs*}

\author{
Seyed Hassan Alavi ${ }^{\dagger}$, Ashraf Daneshkhah, Narges Okhovat \\ Department of Mathematics, Faculty of Science, Bu-Ali Sina University, Hamedan, Iran
}

Received 27 December 2018, accepted 4 November 2019, published online 12 December 2019

\begin{abstract}
In this article, we study flag-transitive automorphism groups of non-trivial symmetric $(v, k, \lambda)$ designs, where $\lambda$ divides $k$ and $k \geqslant \lambda^{2}$. We show that such an automorphism group is either point-primitive of affine or almost simple type, or point-imprimitive with parameters $v=\lambda^{2}(\lambda+2)$ and $k=\lambda(\lambda+1)$, for some positive integer $\lambda$. We also provide some examples in both possibilities.
\end{abstract}

Keywords: Symmetric design, flag-transitive, point-primitive, point-imprimitive, automorphism group. Math. Subj. Class.: 05B05, 05B25, 20B25

\section{Introduction}

A $t$-design $\mathcal{D}=(\mathcal{P}, \mathcal{B})$ with parameters $(v, k, \lambda)$ is an incidence structure consisting of a set $\mathcal{P}$ of $v$ points, and a set $\mathcal{B}$ of $k$-element subsets of $\mathcal{P}$, called blocks, such that every $t$-element subset of points lies in exactly $\lambda$ blocks. The design $\mathcal{D}$ is non-trivial if $t<$ $k<v-t$, and is symmetric if $|\mathcal{B}|=v$. By [7, Theorem 1.1], if $\mathcal{D}$ is symmetric and non-trivial, then $t \leqslant 2$, see also [12, Theorem 1.27]. Thus we study non-trivial symmetric 2 -designs with parameters $(v, k, \lambda)$ which we simply call non-trivial symmetric $(v, k, \lambda)$ designs. A flag of $\mathcal{D}$ is an incident pair $(\alpha, B)$, where $\alpha$ and $B$ are a point and a block of $\mathcal{D}$, respectively. An automorphism of a symmetric design $\mathcal{D}$ is a permutation of the points permuting the blocks and preserving the incidence relation. An automorphism group $G$ of $\mathcal{D}$ is called flag-transitive if it is transitive on the set of flags of $\mathcal{D}$. If $G$ leaves invariant a non-trivial partition of $\mathcal{P}$, then $G$ is said to be point-imprimitive; otherwise $G$ is called

* The authors would like to thank anonymous referees for providing us helpful and constructive comments and suggestions.

†Corresponding author.

E-mail addresses: alavi.s.hassan@basu.ac.ir, alavi.s.hassan@gmail.com (Seyed Hassan Alavi), adanesh@basu.ac.ir, daneshkhah.ashraf@gmail.com (Ashraf Daneshkhah), okhovat.nargeshh@gmail.com (Narges Okhovat) 
point-primitive. We here adopt the standard notation as in [8, 23] for finite simple groups of Lie type. For example, we use $\mathrm{PSL}_{n}(q), \mathrm{PSp}_{n}(q), \mathrm{PSU}_{n}(q), \mathrm{P}_{2 n+1}(q)$ and $\mathrm{P} \Omega_{2 n}^{ \pm}(q)$ to denote the finite classical simple groups. Symmetric and alternating groups on $n$ letters are denoted by $S_{n}$ and $A_{n}$, respectively. Further notation and definitions in both design theory and group theory are standard and can be found, for example in [10, 12, 14]. We also use the software GAP [21] for computational arguments.

Flag-transitive incidence structures have been of most interest. In 1961, Higman and McLaughlin [11] proved that a flag-transitive automorphism group of a linear space must act primitively on its points set, and then Buekenhout, Delandtsheer and Doyen [5] studied this action in details and proved that a linear space admitting a flag-transitive automorphism group (which is in fact point-primitive) is either of affine, or almost simple type. Thereafter, a deep result [6], namely the classification of flag-transitive finite linear spaces relying on the Classification of Finite Simple Groups (CFSG) was announced. Although, flagtransitive symmetric designs are not necessarily point-primitive, Regueiro [18] proved that a flag-transitive and point-primitive automorphism group of such designs for $\lambda \leqslant 4$ is of affine or almost simple type, and so using CFSG, she determined all flag-transitive and point-primitive biplanes $(\lambda=2)$. In conclusion, she gave a classification of flag-transitive biplanes except for the 1-dimensional affine case [17]. Tian and Zhou [22] proved that a flag-transitive and point-primitive automorphism group of a symmetric design with $\lambda \leqslant$ 100 must be of affine or almost simple type. Generally, Zieschang [25] proved in 1988 that a flag-transitive automorphism group of a 2-design with $\operatorname{gcd}(r, \lambda)=1$ is (point-primitive) of affine or almost simple type, and this result has been generalised by Zhuo and Zhan [24] for $\lambda \geqslant \operatorname{gcd}(r, \lambda)^{2}$.

\subsection{Main result}

In this paper, we study flag-transitive automorphism groups of symmetric $(v, k, \lambda)$ designs, where $\lambda$ divides $k$ and $k \geqslant \lambda^{2}$, and we show that such an automorphism group is not necessarily point-primitive:

Theorem 1.1. Let $\mathcal{D}=(\mathcal{P}, \mathcal{B})$ be a non-trivial symmetric $(v, k, \lambda)$ design with $\lambda \geqslant 1$, and let $G$ be a flag-transitive automorphism group of $\mathcal{D}$. If $\lambda$ divides $k$ and $k \geqslant \lambda^{2}$, then one of the following holds:

(a) G is point-primitive of affine or almost simple type;

(b) $G$ is point-imprimitive and $v=\lambda^{2}(\lambda+2)$ and $k=\lambda(\lambda+1)$, for some positive integer $\lambda$. In particular, if $G$ has $d$ classes of imprimitivity of size $c$, then there is a constant $l$ such that, for each block $B$ and each class $\Delta$, the size $|B \cap \Delta|$ is either 0 , or $l$, and $(c, d, l)=\left(\lambda^{2}, \lambda+2, \lambda\right)$ or $\left(\lambda+2, \lambda^{2}, 2\right)$.

We highlight here that if $\lambda$ divides $k$, then $\operatorname{gcd}(k, \lambda)^{2}=\lambda^{2}>\lambda$ which does not satisfy the conditions which have been studied in [24, 25]. Moreover, in Section 1.2, we provide some examples to show that both possibilities in Theorem 1.1 can actually occur.

In order to prove Theorem 1.1(a), we apply O'Nan-Scott Theorem [15] and discuss possible types of primitive groups in Section 3. We further note that our proof for part (a) relies on CFSG. To prove part (b), we use an important result by Praeger and Zhou [20, Theorem 1.1] on characterisation of imprimitive flag-transitive symmetric designs. 


\subsection{Examples and comments on Theorem 1.1}

Here, we give some examples of symmetric $(v, k, \lambda)$ designs admitting flag-transitive automorphism groups, where $\lambda$ divides $k$ and $k \geqslant \lambda^{2}$. In Table 1, we list some small examples of such designs with $\lambda \leqslant 3$. To our knowledge the design in Line 2 is the only point-primitive example of symmetric designs with $v \leqslant 2500$ satisfying the conditions of Theorem 1.1 and this motivates the authors to investigate symmetric designs admitting symplectic automorphism groups [3]. More examples of symmetric designs admitting flagtransitive and point-imprimitive automorphism groups can be found in [20] and references therein.

Line 1. Hussain [13] showed that there are exactly three symmetric $(16,6,2)$ designs, and Regueiro proved that exactly two of such designs are flag-transitive and point-imprimitive [18, p. 139].

Line 2. The symmetric design in this line arises from the study of primitive permutation groups with small degrees. This design belongs to a class of symmetric designs with parameters $\left(3^{m}\left(3^{m}+1\right) / 2,3^{m-1}\left(3^{m}-1\right) / 2,3^{m-1}\left(3^{m-1}-1\right) / 2\right)$, for some positive integer $m>1$, see [4, 9]. If $m=2$, then we obtain the symmetric $(45,12,3)$ design admitting $\mathrm{PSp}_{4}(3)$ or $\mathrm{PSp}_{4}(3): 2$ as flag-transitive automorphism group of rank 3, see [4].

Lines 3-4. Mathon and Spence [16] constructed 2616 pairwise non-isomorphic symmetric $(45,12,3)$ designs with non-trivial automorphism groups. Praeger [19] proved that there are exactly two flag-transitive symmetric $(45,12,3)$ designs, exactly one of which admits a point-imprimitive group, and this example satisfies Line 4, but not Line 3 .

Table 1: Some symmetric designs satisfying the conditions in Theorem 1.1.

\begin{tabular}{crrrrrrrccl} 
Line & $v$ & $k$ & $\lambda$ & $c$ & $d$ & $l$ & Case & Examples & Reference & Comments \\
\hline \hline 1 & 16 & 6 & 2 & 4 & 4 & 2 & (b) & 2 & {$[13],[18]$} & imprimitive \\
2 & 45 & 12 & 3 & - & - & - & (a) & 1 & {$[4]$} & primitive \\
3 & 45 & 12 & 3 & 5 & 9 & 2 & (b) & None & {$[19]$} & imprimitive \\
4 & 45 & 12 & 3 & 9 & 5 & 3 & (b) & 1 & {$[19]$} & imprimitive
\end{tabular}

\section{Preliminaries}

In this section, we state some useful facts in both design theory and group theory.

Lemma 2.1 ([1, Lemma 2.1]). Let $\mathcal{D}$ be a symmetric $(v, k, \lambda)$ design, and let $G$ be a flag-transitive automorphism group of $\mathcal{D}$. If $\alpha$ is a point in $\mathcal{P}$ and $H:=G_{\alpha}$, then

(a) $k(k-1)=\lambda(v-1)$;

(b) $k$ divides $|H|$ and $\lambda v<k^{2}$.

Lemma 2.2 ([2, Corollary 4.3]). Let $T$ be a finite simple classical group of dimension $n$ over a finite field $\mathbb{F}_{q}$ of size $q$. Then 
(a) If $T=\operatorname{PSL}_{n}(q)$ with $n \geqslant 2$, then $|T|>q^{n^{2}-2}$;

(b) If $T=\operatorname{PSU}_{n}(q)$ with $n \geqslant 3$, then $|T|>\left(1-q^{-1}\right) q^{n^{2}-2}$;

(c) If $T=\operatorname{PSp}_{n}(q)$ with $n \geqslant 4$, then $|T|>q^{\frac{1}{2} n(n+1)} /(2 \alpha)$, where $\alpha=\operatorname{gcd}(2, q-1)$;

(d) If $T=\mathrm{P} \Omega_{n}^{\epsilon}(q)$ with $n \geqslant 7$, then $|T|>q^{\frac{1}{2} n(n-1)} /(4 \beta)$, where $\beta=\operatorname{gcd}(2, n)$.

Lemma 2.3. Let $T$ be a non-abelian finite simple group satisfying

$$
|T|<8 \cdot|\operatorname{Out}(T)|^{3}
$$

Then $T$ is isomorphic to $A_{5}$ or $A_{6}$.

Proof. If $T$ is a sporadic simple group or an alternating group $A_{n}$ with $n \geqslant 7$, then $|\operatorname{Out}(T)| \in\{1,2\}$, and so by (2.1), we must have $|T|<64$, which is a contradiction. Note that the alternating groups $A_{5}$ and $A_{6}$ satisfy (2.1) as claimed. Therefore, we only need to consider the case where $T$ is a finite simple group of Lie type. In what follows, we discuss each case separately.

Let $T=\operatorname{PSL}_{n}(q)$ with $q=p^{a}$ and $n \geqslant 2$. If $n=2$, then $q \geqslant 4$ and $|\operatorname{Out}(T)|=$ $a \cdot \operatorname{gcd}(2, q-1)$, and so by Lemma 2.2(a) and (2.1), we have that $q^{2}<\left|\operatorname{PSL}_{2}(q)\right|<$ $8 a^{3} \cdot \operatorname{gcd}(2, q-1)^{3} \leqslant 64 a^{3}$. Thus, $q^{2}<64 a^{3}$. This inequality holds only for $(p, a) \in$ $\{(2,1),(2,2),(2,3),(2,4),(2,5),(2,6),(2,7),(3,1),(3,2),(3,3),(5,1),(7,1)\}$. Note in this case that $q \geqslant 4$, and hence by (2.1), we conclude that $T$ is either

$$
\mathrm{PSL}_{2}(4) \cong \mathrm{PSL}_{2}(5) \cong A_{5}, \quad \text { or } \quad \mathrm{PSL}_{2}(9) \cong A_{6} \text {, }
$$

as claimed. If $n=3$, then by Lemma 2.2(a), we have that $q^{7}<64 a^{3} \cdot \operatorname{gcd}(3, q-1)^{3}<$ $64 a^{3} q^{3}$, and so $q^{4}<64 a^{3}$. If $q$ would be odd, then we would have $3^{4 a}<64 a^{3}$, which is impossible. If $q=2^{a}$, then $2^{a}<64 a^{3}$ would hold only for $a=1,2$. Therefore, $T$ is isomorphic to $\mathrm{PSL}_{3}(2)$ or $\mathrm{PSL}_{3}(4)$. These simple groups do not satisfy (2.1). If $n \geqslant 4$, then (2.1) implies that $q^{11}<64 a^{3}$, but this inequality has no possible solution.

Let $T=\operatorname{PSU}_{n}(q)$ with $q=p^{a}$ and $n \geqslant 3$. By Lemma 2.2(b), we have that $|T|>$ $\left(1-q^{-1}\right) q^{n^{2}-2}$, and so (2.1) implies that $\left(1-q^{-1}\right) q^{n^{2}-2}<64 a^{3} \cdot \operatorname{gcd}(n, q+1)^{3}$. If $n=3$, then $\left(1-q^{-1}\right) q^{7}<64 a^{3} \cdot \operatorname{gcd}(n, q+1)^{3}$, and so $q^{6}<27 \cdot 64 a^{3}$. This inequality holds only for $(p, a) \in\{(2,1),(2,2),(3,1)\}$. Note that $\operatorname{PSU}_{3}(2)$ is not simple. Therefore, $T$ is isomorphic to $\mathrm{PSU}_{3}(3)$ or $\mathrm{PSU}_{3}(4)$. These simple groups do not satisfy (2.1). If $n \geqslant 4$, then since $(q+1)^{3}<4 \cdot q^{3}(q-1)$, we would have $q^{n^{2}-3}<64 a^{3} \cdot \operatorname{gcd}(n, q+1)^{3} /(q-1)<$ $4 \cdot 64 a^{3}(q+1)^{3} / 4(q-1)<4 \cdot 64 a^{3} q^{3}$, and so $q^{n^{2}-6}<4 \cdot 64 a^{3}$, and hence $q^{10}<4 \cdot 64 a^{3}$, which is impossible.

Let $T=\operatorname{PSp}_{n}(q)$ with $q=p^{a}$ and $n \geqslant 4$. By Lemma 2.2(c), we observe that $|T|>$ $q^{\frac{1}{2} n(n+1)} / 2 \operatorname{gcd}(2, q-1) \geqslant q^{\frac{1}{2} n(n+1)} / 4$. By $(2.1)$, we have that $q^{10} \leqslant q^{\frac{1}{2} n(n+1)}<4 \cdot 64 a^{3}$, and so $q^{10}<4 \cdot 64 a^{3}$, which is impossible.

Let $T=\mathrm{P} \Omega_{n}(q)$ with $q=p^{a}$ odd and $n \geqslant 7$. Then we conclude by Lemma 2.2(d) that $|T|>q^{\frac{1}{2} n(n-1)} / 8$. Since $|\operatorname{Out}(T)|=2 a$ and $n \geqslant 7$, it follows from (2.1) that $q^{21}<8^{3} a^{3}$, which is impossible.

Let $T=\mathrm{P} \Omega_{n}^{\epsilon}(q)$ with $q=p^{a}$ and $n \geqslant 8$ and $\epsilon= \pm$. It follows from Lemma 2.2(d) that $|T|>q^{\frac{1}{2} n(n-1)} / 8$. Note that $|\operatorname{Out}(T)| \leqslant 6 a \cdot \operatorname{gcd}\left(4, q^{\frac{n}{2}}-\epsilon\right) \leqslant 24 a$. Then (2.1) implies that $q^{28}<8^{2} \cdot 24^{3} a^{3}$, which is impossible. 
Let $T$ be one of the finite exceptional groups

$$
F_{4}(q), E_{6}(q), E_{7}(q), E_{8}(q),{ }^{2} F_{4}(q)\left(q=2^{2 m+1}\right),{ }^{3} D_{4}(q) \text { and }{ }^{2} E_{6}(q) .
$$

Then $|T|>q^{20}$, and so (2.1) implies that $q^{20}<8 \cdot 2^{3} \cdot 3^{3} a^{3}$, which is impossible. If $T=$ $G_{2}(q)$ with $q=p^{a} \neq 2$. Then by (2.1), we have that $q^{12}<q^{6}\left(q^{2}-1\right)\left(q^{6}-1\right)<8 \cdot 2^{3} a^{3}$, and so $q^{12}<8 \cdot 2^{3} a^{3}$, which is impossible. Similarly, if $T$ is one of the groups ${ }^{2} B_{2}(q)$ with $q=2^{2 m+1}$ and ${ }^{2} G_{2}(q)$ with $q=3^{2 m+1}$, then $|T|>q^{4}$, and so (2.1) implies that $q^{4}<8 a^{3}$, which is impossible.

\section{Point-primitive designs}

In what follows, we assume that $\mathcal{D}=(\mathcal{P}, \mathcal{B})$ is a non-trivial symmetric $(v, k, \lambda)$ design admitting a flag-transitive and point-primitive automorphism group $G$. Let also $\lambda$ divide $k$ and $k \geqslant \lambda^{2}$ and set $t:=k / \lambda$. Notice that $\lambda<k$, and so $t \geqslant 2$. We moreover observe by Lemma 2.1(a) that

$$
\begin{aligned}
& k=\frac{v+t-1}{t} ; \\
& \lambda=\frac{v+t-1}{t^{2}} .
\end{aligned}
$$

Since also $G$ is a primitive permutation group on $\mathcal{P}$, by O'Nan-Scott Theorem [15], $G$ is of one of the following types:

(a) Affine;

(b) Almost simple;

(c) Simple diagonal;

(d) Product;

(e) Twisted wreath product.

\subsection{Product and twisted wreath product type}

In this section, we assume that $G$ is a primitive group of product type on $\mathcal{P}$, that is to say, $G \leqslant H \imath S_{\ell}$, where $H$ is of almost simple or diagonal type on the set $\Gamma$ of size $m:=|\Gamma| \geqslant 5$ and $\ell \geqslant 2$. In this case, $\mathcal{P}=\Gamma^{\ell}$.

Lemma 3.1. Let $G$ be a flag-transitive point-primitive automorphism group of product type. Then $k$ divides $\lambda \ell(m-1)$.

Proof. See the proof of Lemma 4 in [18].

Proposition 3.2. If $\mathcal{D}=(\mathcal{P}, \mathcal{B})$ is a non-trivial symmetric $(v, k, \lambda)$ design admitting a flag-transitive and point-primitive automorphism group $G$, where $\lambda$ divides $k$ and $k \geqslant \lambda^{2}$, then $G$ is not of product type.

Proof. Assume the contrary. Suppose that $G$ is of product type. Then $v=m^{\ell}$. Note by Lemma 3.1 that $k$ divides $\lambda \ell(m-1)$, and so $t=k / \lambda$ divides $\ell(m-1)$. We also note by 
Lemma 2.1(b) that $\lambda v<k^{2}$. Then $v<\lambda t^{2}$, and since $\lambda \leqslant t$, we have that $v<t^{3}$. Recall that $t$ divides $\ell(m-1)$. Hence

$$
m^{\ell}<\ell^{3}(m-1)^{3}
$$

Then $m^{\ell}<\ell^{3} m^{3}$, or equivalently, $m^{\ell-3}<\ell^{3}$. Since $m \geqslant 5$, it follows that $5^{\ell-3}<\ell^{3}$, and this is true for $2 \leqslant \ell \leqslant 6$. If $\ell=6$, then since $m^{6-3}<6^{3}$, we conclude that $m=5$, but $(m, \ell)=(5,6)$ does not satisfy (3.3). Therefore, $2 \leqslant \ell \leqslant 5$.

Suppose first that $\ell=5$. Then by (3.3), we have that $m^{5}<5^{3}(m-1)^{3}$, and so $5 \leqslant m \leqslant 9$. It follows from (3.1) that $t$ divides $m^{5}-1$. For each $5 \leqslant m \leqslant 9$, we can obtain divisors $t$ of $m^{5}-1$. Note by (3.2) that $t^{2}$ must divide $m^{5}-t+1$. This is true only for $m=7$ when $t=2$ or 6 for which

$$
(v, k, \lambda)=(16807,8404,4202) \text { or }(16807,2802,467),
$$

respectively. Since $\lambda^{2} \leqslant k$, these parameters can be ruled out.

Suppose that $\ell=4$. Then by (3.3), we have that $m^{5}<4^{3}(m-1)^{3}$, and so $5 \leqslant m \leqslant 9$. By the same argument as in the case where $\ell=5$, by (3.1) and (3.2), we obtain possible parameters $(m, t, v, k, \lambda)$ as in Table 2 . Note by Lemma 3.1 that $k$ must divide $4 \lambda(m-1)$, and this is not true, for all parameters in Table 2.

Table 2: Possible values for $(m, t, v, k, \lambda)$ when $\ell=4$.

\begin{tabular}{rrrrr}
$m$ & $t$ & $v$ & $k$ & $\lambda$ \\
\hline \hline 13 & 51 & 28561 & 561 & 11 \\
31 & 555 & 923521 & 1665 & 3 \\
47 & 345 & 4879681 & 14145 & 41 \\
57 & 416 & 10556001 & 25376 & 61
\end{tabular}

Suppose now that $\ell=3$. We again apply Lemma 3.1 and conclude that $t$ divides $3(m-1)$. Then there exists a positive integer $x$ such that $3(m-1)=t x$, and so $m=$ $(t x+3) / 3$. By (3.2), we have that

$$
\lambda=\frac{m^{2}+t-1}{t^{2}}=\frac{t^{2} x^{3}+9 t x^{2}+27 x+27}{27 t} .
$$

Then $27 \lambda t=t^{2} x^{3}+9 t x^{2}+27 x+27$. Therefore, $t$ must divide $27 x+27$, and so $t y=$ $27 x+27$, for some positive integer $y$. Thus,

$$
\lambda=\frac{t(t y-27)^{3}+9 \cdot 27(t y-27)^{2}+27^{3} y}{27^{4}},
$$

for some positive integers $t$ and $y$. Since $\lambda^{2} \leqslant k$, we have that $\lambda \leqslant t$, and so

$$
t(t y-27)^{3}+9 \cdot 27(t y-27)^{2}+27^{3} y \leqslant 27^{4} t .
$$

If $y \geqslant 32$, then

$$
\begin{aligned}
t(t y-27)^{3}+ & 9 \cdot 27(t y-27)^{2}+27^{3} y \\
& \geqslant t(32 t-27)^{3}+9 \cdot 27(32 t-27)^{2}+32 \cdot 27^{3}>27^{4} t
\end{aligned}
$$


for $t \geqslant 2$. Thus $1 \leqslant y \leqslant 31$, and so by (3.5), we conclude that $2 \leqslant t \leqslant 107$. For each such $y$ and $t$, by straightforward calculation, we observe that $\lambda$ as in (3.4) is not a positive integer.

Suppose finally that $\ell=2$. Recall by Lemma 3.1 that $t$ divides $2(m-1)$. Then $2(m-1)=t x$ for some positive integer $x$, and so $m=(t x+2) / 2$. It follows from (3.2) that $\lambda=\left(t x^{2}+4 x+4\right) / 4 t$, or equivalently, $4 t \lambda=t x^{2}+4 x+4$. This shows that $t$ divides $4 x+4$, and so $t y=4 x+4$, for some positive integer $y$. Therefore, $4^{3} \lambda=(t y-4)^{2}+16 y$. Since $\lambda^{2} \leqslant k$, we have that $\lambda \leqslant t$, and so $(t y-4)^{2}+16 y \leqslant 4^{3} t$. If $y \geqslant 6$, then $(6 t-4)^{2}+6 \cdot 16 \leqslant 4^{3} t$, which has no possible solution for $t$. Thus $1 \leqslant y \leqslant 5$. Since also $(t-4)^{2}+16 \leqslant 4^{3} t$, we conclude that $2 \leqslant t \leqslant 71$, and so (3.1) and (3.2) imply that

$$
k=\frac{t\left(t^{2} y^{2}-8 t y+16 y+16\right)}{64} \quad \text { and } \quad \lambda=\frac{(t y-4)^{2}+16 y}{64},
$$

where $2 \leqslant t \leqslant 71$ and $1 \leqslant y \leqslant 5$. For these values of $t$ and $y$, considering the fact that $m \geqslant 5, k \geqslant \lambda^{2}$ and $\lambda$ divides $k$, we obtain $(v, k, \lambda)=(121,25,5)$ or $(441,56,7)$ respectively when $(t, y)=(5,4)$ or $(8,3)$. These possibilities can be ruled out by [4] or [22, Theorem 1.1].

Proposition 3.3. If $\mathcal{D}=(\mathcal{P}, \mathcal{B})$ is a non-trivial symmetric $(v, k, \lambda)$ design admitting a flag-transitive and point-primitive automorphism group $G$, where $\lambda$ divides $k$ and $k \geqslant \lambda^{2}$, then $G$ is not of twisted wreath product type.

Proof. If $G$ would be of twisted wreath product type, then by [15, Remark 2(ii)], it would be contained in the wreath product $H \imath S_{m}$ with $H=T \times T$ of simple diagonal type, and so $G$ would act on $\mathcal{P}$ by product action, and this contradicts Proposition 3.2.

\subsection{Simple diagonal type}

In this section, we suppose that $G$ is a primitive group of diagonal type. Let $M=$ $\operatorname{Soc}(G)=T_{1} \times \cdots \times T_{m}$, where $T_{i} \cong T$ is a non-abelian finite simple group, for $i=1, \ldots, m$. Then $G$ may be viewed as a subgroup of $M \cdot\left(\operatorname{Out}(T) \times S_{m}\right)$. Here, $G_{\alpha}$ is isomorphic to a subgroup of $\operatorname{Aut}(T) \times S_{m}$ and $M_{\alpha} \cong T$ is a diagonal subgroup of $M$, and so $|\mathcal{P}|=|T|^{m-1}$.

Lemma 3.4. Let $G$ be a flag-transitive point-primitive automorphism group of simple diagonal type with socle $T^{m}$. Then $k$ divides $\lambda m_{1} h$, where $m_{1} \leqslant m$ and $h$ divides $|T|$.

Proof. See the proof of Proposition 3.1 in [22].

Proposition 3.5. If $\mathcal{D}=(\mathcal{P}, \mathcal{B})$ is a non-trivial symmetric $(v, k, \lambda)$ design admitting a flag-transitive and point-primitive automorphism group $G$, where $\lambda$ divides $k$ and $k \geqslant \lambda^{2}$, then $G$ is not of simple diagonal type.

Proof. Suppose by contradiction that $G$ is a primitive group of simple diagonal type. Then $v=|T|^{m-1}$, and so by Lemma 2.1(b), $\lambda v<k^{2}$. This implies that $\lambda|T|^{m-1}<k^{2}=\lambda^{2} t^{2}$. Since $\lambda^{2} \leqslant k$, we must have $\lambda \leqslant t$, and hence

$$
|T|^{m-1}<t^{3}
$$

Note by Lemma 3.4 that $k$ divides $\lambda m_{1} h$ and $m_{1} h \leqslant m|T|$. Then $t$ divides $m_{1} h$, and so $t \leqslant m|T|$. We now apply (3.6) and conclude that $|T|^{m-1}<m^{3}|T|^{3}$. Therefore, 
$|T|^{m-4}<m^{3}$. Since $|T| \geqslant 60$, we must have $m<6$. If $m=5$, then $|T|<5^{3}$, and it follows that $T \cong A_{5}$. Note that $k$ divides $\lambda(v-1)=\lambda\left(|T|^{m-1}-1\right)$. Then $t$ divides $|T|^{m-1}-1=60^{4}-1=13 \cdot 59 \cdot 61 \cdot 277$. Since $t \leqslant m|T|=300$ and $t \geqslant 2$, it follows that $t \in\{13,59,61,277\}$. For each such $t$, we have that $\lambda \leqslant t$ and $k=t \lambda$, and so we easily observe that these parameters does not satisfy Lemma 2.1(a). Therefore $m \in\{2,3,4\}$. Note that $G_{\alpha}$ is isomorphic to a subgroup of $\operatorname{Aut}(T) \times S_{m}$. Then by Lemma 2.1(b), the parameter $k$ divides $\left|G_{\alpha}\right|$, and so $k$ divides $(m !) \cdot|T| \cdot|\operatorname{Out}(T)|$. On the other hand, Lemma 2.1(a) implies that $k$ divides $\lambda\left(|T|^{m-1}-1\right)$, and so $t$ divides $|T|^{m-1}-1$ implying that $\operatorname{gcd}(t,|T|)=1$. Since $k$ divides $(m !) \cdot|T| \cdot|\operatorname{Out}(T)|$ and $t$ is a divisor of $k$, we conclude that $t$ divides $(m !) \cdot|\operatorname{Out}(T)|$. Recall by (3.6) that $|T|^{m-1}<t^{3}$. Therefore,

$$
|T|^{m-1}<(m !)^{3} \cdot|\operatorname{Out}(T)|^{3},
$$

where $m \in\{2,3,4\}$.

If $m=2$, then $|T|<8 \cdot|\operatorname{Out}(T)|^{3}$. If $m=3$, then $|T|^{2}<6^{3}|\operatorname{Out}(T)|^{3}$, and so $|T|<6^{\frac{3}{2}}|\operatorname{Out}(T)|$. If $m=4$, then $|T|^{3}<24^{3}|\operatorname{Out}(T)|^{3}$, and $|T|<24|\operatorname{Out}(T)|$. Thus for $m \leqslant 4$, we always have

$$
|T|<8 \cdot|\operatorname{Out}(T)|^{3}
$$

where $T$ is a non-abelian finite simple group. We now apply Lemma 2.3 and conclude that $T$ is isomorphic to $A_{5}$ or $A_{6}$. If $m=2$, then since $t$ divides $|T|^{m-1}-1=|T|-1$, we have that $t$ divides 59 or 359 when $T$ is isomorphic to $A_{5}$ or $A_{6}$, respectively. Thus $(v, k, \lambda)=(60,59 \lambda, \lambda)$ or $(v, k, \lambda)=(360,359 \lambda, \lambda)$. Since $\lambda>1$, in each case, we conclude that $k>v$, which is a contradiction. For $m=3,4$, since $\left|\operatorname{Out}\left(A_{5}\right)\right|=2$ and $\left|\operatorname{Out}\left(A_{6}\right)\right|=4$, it follows from (3.7) that $|T|<48$ or $|T|<96$ when $T$ is isomorphic to $A_{5}$ or $A_{6}$, respectively, which is a contradiction.

\section{Proof of the main result}

In this section, we prove Theorem 1.1. Suppose that $\mathcal{D}=(\mathcal{P}, \mathcal{B})$ is a non-trivial symmetric $(v, k, \lambda)$ design with $\lambda$ divides $k$ and $k \geqslant \lambda^{2}$. Suppose also that $G$ is a flag-transitive automorphism group of $\mathcal{D}$.

Proof of Theorem 1.1. If $G$ is point-primitive, then by O'Nan-Scott Theorem [15] and Propositions 3.2, 3.3 and 3.5, we conclude that $G$ is of affine or almost simple type. Suppose now that $G$ is point-imprimitive. Then $G$ leaves invariant a non-trivial partition $\mathcal{C}$ of $\mathcal{P}$ with $d$ classes of size $c$. By [20, Theorem 1.1], there is a constant $l$ such that, for each $B \in \mathcal{B}$ and $\Delta \in \mathcal{C},|B \cap \Delta| \in\{0, l\}$ and one of the following holds:

(a) $k \leqslant \lambda(\lambda-3) / 2$;

(b) $(v, k, \lambda)=\left(\lambda^{2}(\lambda+2), \lambda(\lambda+1), \lambda\right)$ with $(c, d, l)=\left(\lambda^{2}, \lambda+2, \lambda\right)$ or $\left(\lambda+2, \lambda^{2}, 2\right)$;

(c)

$$
(v, k, \lambda, c, d, l)=\left(\frac{(\lambda+2)\left(\lambda^{2}-2 \lambda+2\right)}{4}, \frac{\lambda^{2}}{2}, \lambda, \frac{\lambda+2}{2}, \frac{\lambda^{2}-2 \lambda+2}{2}, 2\right),
$$

and either $\lambda \equiv 0(\bmod 4)$, or $\lambda=2 u^{2}$, where $u$ is odd, $u \geqslant 3$, and $2\left(u^{2}-1\right)$ is a square; 
(d)

$$
(v, k, \lambda, c, d, l)=\left(\frac{(\lambda+6)\left(\lambda^{2}+4 \lambda-1\right)}{4}, \frac{\lambda(\lambda+5)}{2}, \lambda, \lambda+6, \frac{\lambda^{2}+4 \lambda-1}{4}, 3\right),
$$

where $\lambda \equiv 1$ or $3(\bmod 6)$.

We easily observe that the cases (a) and (c) can be ruled out as $k \geqslant \lambda^{2}$. If case (d) occurs, then $\lambda(\lambda+5) / 2=k \geqslant \lambda^{2}$ implying that $\lambda \leqslant 5$. Since $\lambda \equiv 1$ or $3(\bmod 6)$, it follows that $\lambda=3$ for which $(v, k, \lambda, c, d, l)=(45,12,3,9,5,3)$ which satisfies the condition in Theorem 1.1(b). Therefore, the case (b) can occur as claimed.

\section{References}

[1] S. H. Alavi, M. Bayat and A. Daneshkhah, Symmetric designs admitting flag-transitive and point-primitive automorphism groups associated to two dimensional projective special groups, Des. Codes Cryptogr. 79 (2016), 337-351, doi:10.1007/s10623-015-0055-9.

[2] S. H. Alavi and T. C. Burness, Large subgroups of simple groups, J. Algebra 421 (2015), 187 233, doi:10.1016/j.jalgebra.2014.08.026.

[3] S. H. Alavi, A. Daneshkhah and N. Okhovat, Symmetric designs admitting flag-transitive and point-primitive automorphism groups associated to four dimensional symplectic groups, in preparation.

[4] S. Braić, A. Golemac, J. Mandić and T. Vučičić, Primitive symmetric designs with up to 2500 points, J. Combin. Des. 19 (2011), 463-474, doi:10.1002/jcd.20291.

[5] F. Buekenhout, A. Delandtsheer and J. Doyen, Finite linear spaces with flag-transitive groups, J. Comb. Theory Ser. A 49 (1988), 268-293, doi:10.1016/0097-3165(88)90056-8.

[6] F. Buekenhout, A. Delandtsheer, J. Doyen, P. B. Kleidman, M. W. Liebeck and J. Saxl, Linear spaces with flag-transitive automorphism groups, Geom. Dedicata 36 (1990), 89-94, doi:10. 1007/bf00181466.

[7] A. R. Camina, A survey of the automorphism groups of block designs, J. Combin. Des. 2 (1994), 79-100, doi:10.1002/jcd.3180020205.

[8] J. H. Conway, R. T. Curtis, S. P. Norton, R. A. Parker and R. A. Wilson, Atlas of Finite Groups: Maximal Subgroups and Ordinary Characters for Simple Groups, Oxford University Press, Eynsham, 1985, http://brauer.maths.qmul.ac.uk/Atlas/.

[9] U. Dempwolff, Primitive rank 3 groups on symmetric designs, Des. Codes Cryptogr. 22 (2001), 191-207, doi:10.1023/a:1008373207617.

[10] J. D. Dixon and B. Mortimer, Permutation Groups, volume 163 of Graduate Texts in Mathematics, Springer-Verlag, New York, 1996, doi:10.1007/978-1-4612-0731-3.

[11] D. G. Higman and J. E. McLaughlin, Geometric ABA-groups, Illinois J. Math. 5 (1961), 382 397, doi:10.1215/ijm/1255630883.

[12] D. R. Hughes and F. C. Piper, Design Theory, Cambridge University Press, Cambridge, 2nd edition, 1988.

[13] Q. M. Hussain, Symmetrical incomplete block designs with $\lambda=2, k=8$ or 9 , Bull. Calcutta Math. Soc. 37 (1945), 115-123.

[14] E. S. Lander, Symmetric Designs: An Algebraic Approach, volume 74 of London Mathematical Society Lecture Note Series, Cambridge University Press, Cambridge, 1983, doi: $10.1017 /$ cbo9780511662164. 
[15] M. W. Liebeck, C. E. Praeger and J. Saxl, On the O'Nan-Scott theorem for finite primitive permutation groups, J. Austral. Math. Soc. Ser. A 44 (1988), 389-396, doi:10.1017/ s144678870003216x.

[16] R. Mathon and E. Spence, On 2-(45, 12,3) designs, J. Combin. Des. 4 (1996), 155-175, doi: 10.1002/(sici)1520-6610(1996)4:3〈155::aid-jcd1〉3.3.co;2-1.

[17] E. O'Reilly-Regueiro, Flag-Transitive Symmetric Designs, Ph.D. thesis, University of London, 2003, https://www. matem. unam.mx/ eugenia/papers/thesis.pdf.

[18] E. O'Reilly-Regueiro, On primitivity and reduction for flag-transitive symmetric designs, $J$. Comb. Theory Ser. A 109 (2005), 135-148, doi:10.1016/j.jcta.2004.08.002.

[19] C. E. Praeger, The flag-transitive symmetric designs with 45 points, blocks of size 12 , and 3 blocks on every point pair, Des. Codes Cryptogr. 44 (2007), 115-132, doi:10.1007/ s10623-007-9071-8.

[20] C. E. Praeger and S. Zhou, Imprimitive flag-transitive symmetric designs, J. Comb. Theory Ser. A 113 (2006), 1381-1395, doi:10.1016/j.jcta.2005.12.006.

[21] The GAP Group, GAP - Groups, Algorithms, and Programming, Version 4.7.9, 2015, http : //www.gap-system.org/.

[22] D. Tian and S. Zhou, Flag-transitive point-primitive symmetric $(v, k, \lambda)$ designs with $\lambda$ at most 100, J. Combin. Des. 21 (2013), 127-141, doi:10.1002/jcd.21337.

[23] R. A. Wilson, The Finite Simple Groups, volume 251 of Graduate Texts in Mathematics, Springer-Verlag, London, 2009, doi:10.1007/978-1-84800-988-2.

[24] S. Zhou and X. Zhan, Flag-transitive automorphism groups of 2-designs with $\lambda \geq(r, \lambda)^{2}$ and an application to symmetric designs, Ars Math. Contemp. 14 (2018), 187-195, doi:10.26493/ 1855-3974.1165.105.

[25] P.-H. Zieschang, Flag transitive automorphism groups of 2-designs with $(r, \lambda)=1$, J. Algebra 118 (1988), 369-375, doi:10.1016/0021-8693(88)90027-0. 